\title{
Effect of in-medium properties on heavy-ion collisions
}

\author{
Jürgen Schaffner-Bielich䀦 \\ † RIKEN BNL Research Center, Brookhaven National Laboratory, Upton, NY \\ 11973, USA
}

\begin{abstract}
The properties of strange hadrons, i.e. of kaons and hyperons, in the nuclear medium are discussed in connection with neutron star properties and relativistic heavy-ion collisions. Firstly, the relevant medium modifications of a kaon in a medium as provided by heavy-ion collisions is critically examined within a coupled channel calculation. We demonstrate, that particle ratios for kaons are not a sensitive probe of in-medium effects while the $\mathrm{K}^{-}$flow is more suited to pin down the $\mathrm{K}^{-}$optical potential in dense matter. Secondly, the interaction between hyperons is studied and may form bound states which can be produced in relativistic heavy-ion collisions. Signals for the detection of strange dibaryons by their decay topology and/or in the invariant mass spectra are outlined.
\end{abstract}

\section{Introduction}

We study in the following the in-medium effects of hadrons with strangeness. The paper is split in two main parts, one dealing with kaons and antikaons, the other with hyperons. In both parts, we discuss first our basic knowledge of the underlying kaon/antikaon-nucleon, hyperon-nucleon and hyperon-hyperon interaction, respectively, and how it relates to an optical potential at normal nuclear density. Implications for the properties of neutron stars are discussed. Then we proceed to apply the possible in-medium effects to heavy-ion collisions. In the first part about kaons and antikaons, special emphasis will be put on the fact, that the in-medium effects probed in heavy-ion collisions are entirely different from the ones in the interior of neutron stars. This results in a reinterpretation of the subthreshold production rates of antikaons as measured at GSI. In the second part about hyperons, we focus on the possibility of forming bound states of hyperons in heavy-ion collisions at freeze-out by virtue of a highly attractive hyperon-hyperon interaction. The properties and decay patterns of these strange exotic particles are quite unique and make them well distinguishable experimentally from ordinary hadrons and nuclei.

\section{Kaons in matter}

\subsection{Kaon-nucleon interaction}

The study of kaons in dense matter has attracted much interest in recent years in particular due to its possible implications for neutron stars. For the $\mathrm{K}^{+}$we know 
from the low-density theorem, that the optical potential is repulsive in matter

$$
\Pi_{K^{+}}=t_{K^{+} N} \cdot \rho_{N} \longrightarrow U_{\mathrm{opt}}^{K^{+}}=+30 \mathrm{MeV} \cdot \rho / \rho_{0}
$$

where $t_{K+N}$ is the (known) elementary $\mathrm{K}^{+} \mathrm{N}$ scattering amplitude. The low-density theorem can not be applied to the $\mathrm{K}^{-}$, because of the appearance of inelastic channels in dense matter as $\mathrm{K}^{-} \mathrm{N} \rightarrow \Sigma \pi$. The strong coupling to these inelastic channels, they are about $100 \mathrm{MeV}$ below threshold, gives rise to a large imaginary part and to a resonant state just below threshold so that the isospin zero scattering length is repulsive. This resonant state, the $\Lambda(1405)$ resonance, controls the in-medium properties of the $\mathrm{K}^{-}$. There are two indications, that the optical potential for the $\mathrm{K}^{-}$ changes sign in the medium. Firstly, the study of kaonic atoms reveals that a fit with a nonlinear density dependence can reduce the energy of the kaon by nearly $200 \mathrm{MeV}$ inside the nucleus [1, 2]. Secondly, a coupled channel calculation for the $\mathrm{K}^{-}$in the nuclear medium produces also an attractive potential of $U_{K^{-}} \approx-100 \mathrm{MeV}$ at $\rho_{0}$ [3], although it is much shallower than the one extracted from kaonic atoms. Here, the $\Lambda(1405)$ mode is shifted up in the medium, so that the $\mathrm{K}^{-}$feels its truly attractive potential. The implications for the properties of neutron stars can be quite drastic. Ignoring the hyperon degrees of freedom, the maximum mass of a neutron star is considerably reduced , Als. Also, the minimum radius can decrease from values around $12 \mathrm{~km}$ to nearly $8 \mathrm{~km}[5]$. These small radii occur only for relatively large values of the $\mathrm{K}^{-}$optical potential which are between the values quoted from kaonic atoms and coupled channel calculations. In this case, a first order phase transition occurs in the interior of the neutron star building a mixed phase of an ordinary nucleonic phase and a kaon condensed phase. The smallness of the radius of a neutron star might then signal a first order phase transition. But as we will see below, this is not a unique feature for kaon condensation inside neutron stars.

Heavy-ion collisions provide an important tool to pin down the possible inmedium effects for antikaons. Especially, the production rates of $\mathrm{K}^{-}$at subthreshold bombarding energies are regarded as a measure of the antikaon optical potential [4]. Now the situation in heavy-ion collisions is completely different compared to kaonic atoms or neutron stars. In the latter two cases, the antikaon optical potential is probed at zero momentum and zero temperature. In heavy-ion collisions, on the contrary, matter is in motion and antikaons are produced with a finite momentum relative to the matter rest frame. In addition, the matter is not only dense but also heated up - slope parameters of $90 \mathrm{MeV}$ have been extracted from the hadron spectra. So the relevant observable in heavy-ion collisions is the optical potential of the $\mathrm{K}^{-}$at finite relative momentum and finite temperature.

\subsection{Coupled channel calculations at finite relative momentum and temperature}

We studied the properties of the $\mathrm{K}^{-}$in such an environment within a coupled channel calculation [6]. A simple separable $\mathrm{SU}(3)$ symmetric potential is used whose parameters are fixed by the shape of the $\Lambda(1405)$ resonance. This also gives a reasonable description of the various cross sections, although we underestimate the elastic channel and overestimate the charge exchange reaction $\mathrm{K}^{-} \mathrm{p} \rightarrow \mathrm{K}^{0} \mathrm{n}$. In the medium, the loop integral over the intermediate states $(\bar{K} N, \pi \Sigma, \pi \Lambda)$ is modified. In the following, we just discuss the simplest case, when only the nucleon propagator is changed in the medium. In-medium effects for the hyperons are found to be negligible [6]. A selfconsistent treatment of the antikaon, as done in [7, 8], would only strengthen our conclusions about the optical potential of the antikaon in heavy-ion matter. 


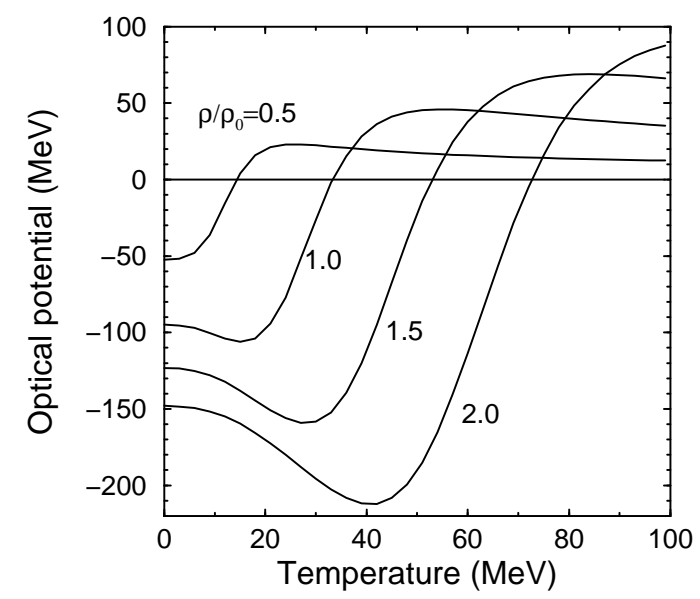

Figure 1. The optical potential of the $\mathrm{K}^{-}$as a function of temperature for different densities (taken from [).

The temperature dependence of the $\mathrm{K}^{-}$optical potential is shown in figure 1 for different densities. One sees, that the $\mathrm{K}^{-}$optical potential suddenly changes sign for a sufficiently large temperature. For normal nuclear density $\rho_{0}$, this happens to be at a critical temperature of $T_{c}=32 \mathrm{MeV}$. For twice normal nuclear density the critical temperature is about twice this value. In both cases, the critical temperature stays well below the slope parameter of $T=90 \mathrm{MeV}$ measured in the relevant heavy-ion experiments at GSI. Note, that the value of $T_{c}$ is equal to the nucleon Fermi energy. Pauli blocking effects begin to wash out and to get ineffective at $T_{c}$ for providing an attractive potential for the antikaon. At large temperatures, the optical potential saturates at a positive (repulsive) value. Here, the interaction between $\mathrm{K}^{-}$and the nucleons can again be described by the low density theorem as the systems gets diluted by the high temperature. The repulsive potential at large temperatures is then just proportional to the repulsive scattering length times the density, as can be read off from figure 1. We found a similar but less pronounced effect, when incorporating a finite relative momentum for the $\mathrm{K}^{-}$optical potential [6. For momenta relevant to subthreshold production of $\mathrm{K}^{-}$at GSI, the optical potential turns out to be repulsive, too. We conclude at his point, that the $\mathrm{K}^{-}$feels a much shallower if not repulsive potential in heavy-ion collisions. Hence, the hitherto used argumentation that a reduced effective energy of the $\mathrm{K}^{-}$is responsible for the enhanced subthreshold production of $\mathrm{K}^{-}$seen at GSI should at least be reconsidered.

There are two comments and a question in order at this point. Firstly, a selfconsistent treatment of the $\mathrm{K}^{-}$results also in much shallower optical potentials of -30 to $-40 \mathrm{MeV}$ at $\rho_{0}$ even for the zero momentum, zero temperature case [7, 8, 6] strengthening our conclusions made above. Recently, a selfconsistent calculation has been performed by Tolos et al within the Bonn model, which takes into account all higher partial waves [9]. They find a value of $-80 \mathrm{MeV}$ for the antikaon optical potential at $\rho_{0}$, which is still lower than the $U_{K^{-}}=-100 \mathrm{MeV}$ of the nonrelativistic coupled channel calculation used above. Secondly, the results for finite momenta have to be taken with care, as p-wave contributions might become important, which have not been included here (see 110]). Again the momentum dependence is much weaker in the Bonn model calculation including all partial waves [9] than in our 


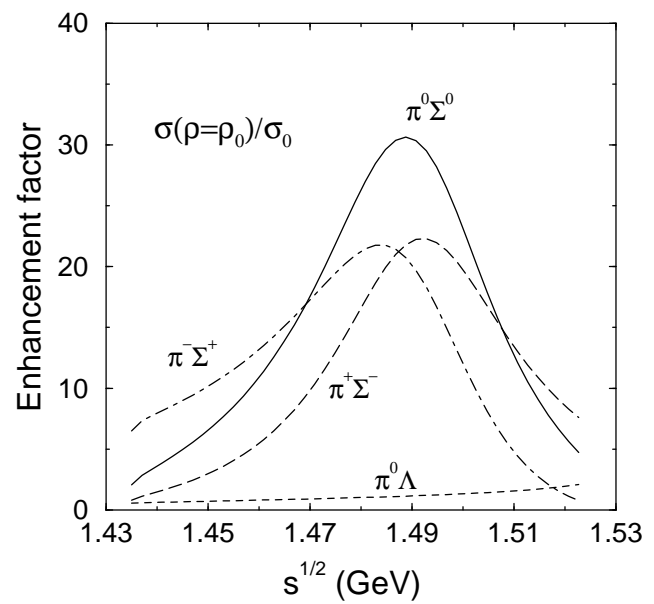

Figure 2. The enhancement of the production cross section of $\mathrm{K}^{-}$at normal nuclear density compared to the zero density case (taken from [6]).

calculations which takes into account only the s-wave contributions. Note, that the large temperature effects seen in figure 11 are unaffected by these comments.

So if the antikaon feels that weak attraction (if at all) in heavy-ion collisions, how can one explain the enhancement of $\mathrm{K}^{-}$production rates as measured at GSI?

One possible explanation might be provided by studying the in-medium cross sections for the antikaons. Indeed, the fact that the $\Lambda(1405)$ mode is shifted up in the medium directly relates to an tremendously enhanced imaginary scattering amplitude, i.e. an enhanced cross section, at and above the threshold energy. We define an enhancement factor of the cross section by the ratio of the cross section at normal nuclear density over the one at zero density. The enhancement factor for the production cross section of the $\mathrm{K}^{-}$via the hyperon-pion channels is depicted in figure 2 versus the colliding energy of the $\mathrm{K}^{-} \mathrm{p}$ pair. The production cross section $\Sigma \pi \rightarrow K^{-} p$ is enhanced by an order of magnitude, while the $\pi^{0} \Lambda$ channel is barely affected. The reason is, that only the isospin zero amplitude, where the $\Lambda(1405)$ is sitting, is enhanced in the medium. The $\pi^{0} \Lambda$ does not couple to isospin zero, while the $\Sigma \pi$ does. This explains the difference in the enhancement factor seen in figure 2. The dominant peak structure around $\sqrt{s}=1.49 \mathrm{GeV}$ stems from the $\Lambda(1405)$ which is shifted well above threshold in the dense nuclear medium. The position of the peak depends on the density. It is noteworthy, that especially the $\pi^{-} \Sigma^{+}$production channel is enhanced at threshold. Indications of this medium enhancement can be deduced from $\mathrm{K}^{-}$absorption on nuclei [11]. As shown in [6], finite momentum, finite temperature and selfconsistency effects are not as crucial for the cross sections as for the optical potential, so that an enhancement, although somehow diminished, prevails. Hence, the cross section enhancement seems to be a viable tool to explain the $\mathrm{K}^{-}$enhancement. As we will see in the following, this is not yet the full story.

\subsection{Confrontation with experimental data on $K^{-}$production}

To elucidate the possible influences of in-medium potentials and in-medium cross sections, a relativistic transport model has to be asked for advice. Here we use the BUU transport model of [12, 13]. Details of the included strangeness production and 


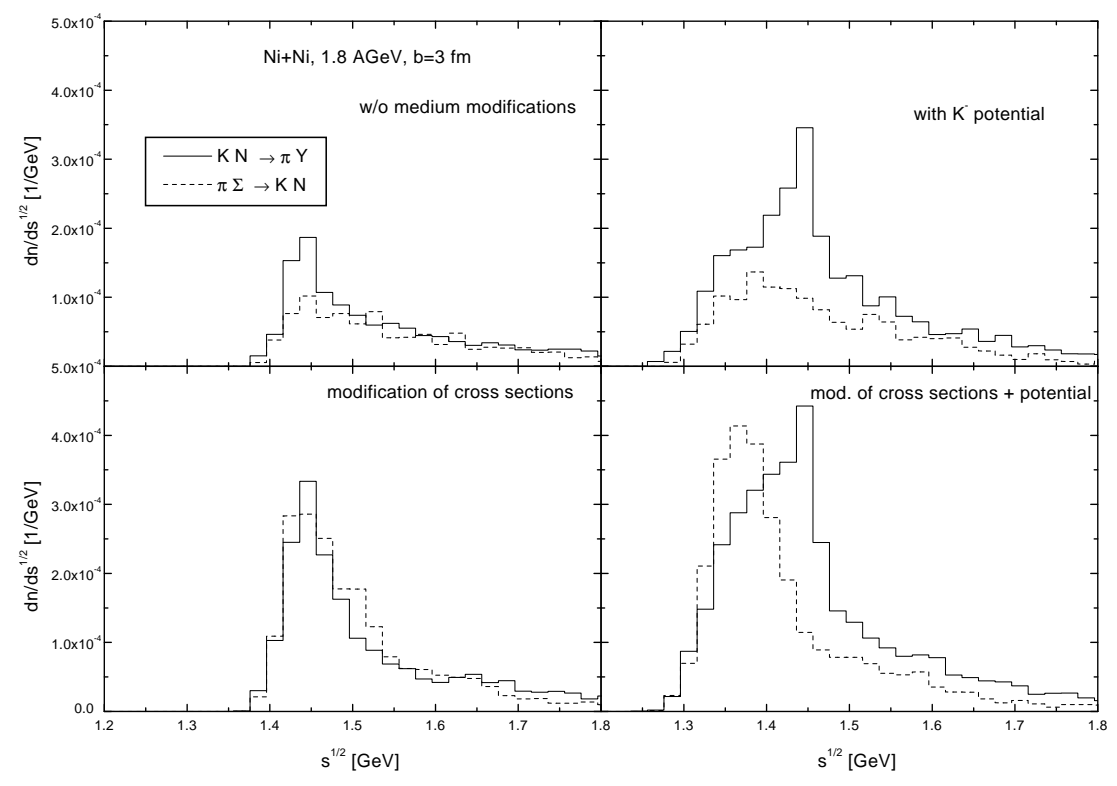

Figure 3. The collisions spectra for the different versions of the in-medium changes for the $\mathrm{K}^{-}$(taken from [6]). The collision rate increases considerably when including effects from the medium modified cross sections.

absorption channels as well as of the implemented kaon-nucleon interactions can be found in [6]. The model gives a reasonable description of the pion and $\mathrm{K}^{+}$production data. For the $\mathrm{K}^{-}$, we implement the following three in-medium effects of our coupled channel calculations in addition to the free case. In all these cases, effects from a finite relative momentum and finite temperature are ignored to study the maximum possible in-medium effect in our approach. Firstly, an optical potential of the $\mathrm{K}^{-}$is included which scales with density as done in all previous simulations with in-medium effects for the $\mathrm{K}^{-}$. Note, that this provides the maximal in-medium effect for the $\mathrm{K}^{-}$optical potential which, as outlined before, is probably weakened at finite relative momentum. Secondly, the in-medium cross section is taken into account. Thirdly, both effects are added to the simulation. This case gives the maximum in-medium effect.

Let us begin by looking at the collision spectra in figure 3. An in-medium potential for the $\mathrm{K}^{-}$increases the number of $\bar{K} N \rightarrow Y \pi$ collisions by about a factor two as more antikaons are produced. The energy shift of the antikaon in the medium also populates the collisions spectra below the threshold energy. A modification of the cross section in the medium triples the number of $\pi \Sigma \rightarrow \bar{K} N$ processes. Finally, the combined effects of in-medium potential and cross section enhances the collisions spectra by a factor five below $\sqrt{s}=1.52 \mathrm{GeV}$. Note, that a modified cross section enhances both reactions, the production as well as the absorption reactions, due to detailed balance. As the systems is close to equilibrium, the enhanced production of antikaons is nearly compensated by the increased absorption rate.

This effect shows up also in the final production cross section (see figure (1). The medium effects for the $\mathrm{K}^{-}$are less drastic than expected from the sizable inmedium changes of the optical potential and the cross sections. We see a maximum enhancement of about a factor of three for low momentum $\mathrm{K}^{-}$compared to the free case which is much less than the anticipated order of magnitude enhancement from the 


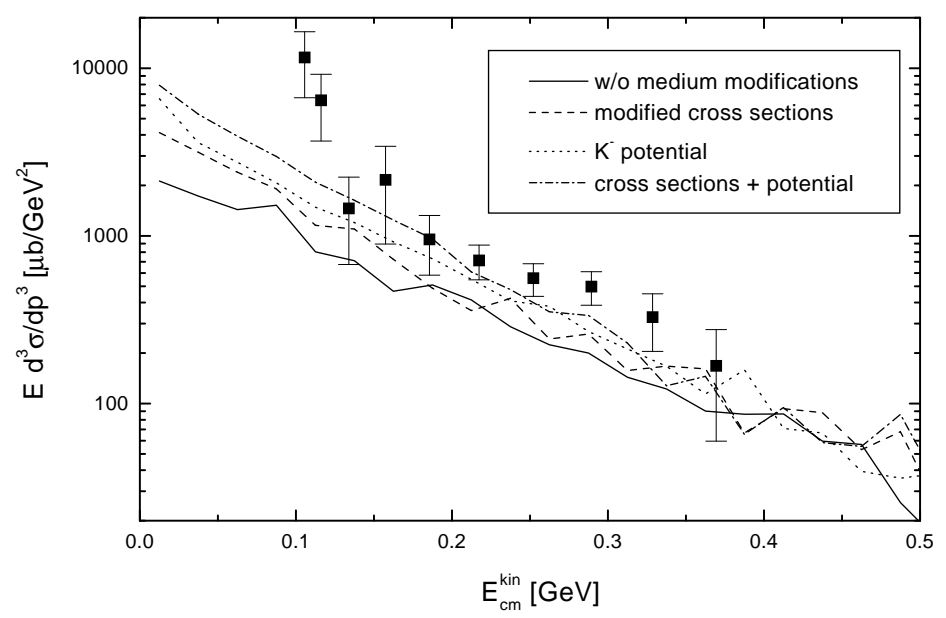

Figure 4. The transverse spectra of the $\mathrm{K}^{-}$for $\mathrm{Ni}+\mathrm{Ni}$ collisions at $1.8 \mathrm{AGeV}$ for various choices of medium modifications (taken from [6]). The data is from the KaoS collaboration 14 .

in-medium cross section enhancement or the optical potential. Interestingly, the result without in-medium effects is already close to the data points of the KaoS collaboration and the inclusion of in-medium effects just puts the curve closer to the data.

The ratio of produced $\mathrm{K}^{-}$to $\mathrm{K}^{+}$as measured by the FOPI collaboration is plotted in figure 5. Again, one notices that the production rate of $\mathrm{K}^{-}$is not very sensitive to in-medium effects. Only the combined maximum effect of a deeply attractive optical potential of the $\mathrm{K}^{-}$and an enhanced cross sections results in a significant deviation of the $\mathrm{K}^{-} / \mathrm{K}^{+}$ratio from the one of the free case. While the free case is on top of the data points, the in-medium effects seem to overpredict the ratio slightly. The in-medium results do not differ significantly from the free case, because the system is already so close to chemical equilibrium that an acceleration of the reaction rates due to larger cross sections or reduced masses has no further influence on the final production yields.

Our results are in accord with the success of the statistical model in the canonical ensemble in describing the kaon and antikaon production data 116, 17]. In this model, no in-medium effect is required to get the $\mathrm{K}^{-} / \mathrm{K}^{+}$ratio, too. Another hint for the success of the statistical model might be provided by the collision spectra of figure 3. The in-medium cross sections enhance the number of collisions involving the $\mathrm{K}^{-}$ without changing the final number considerably, so that a purely statistical analysis becomes applicable. Hence, we judge from figures 14 and 5 that the $\mathrm{K}^{-}$production numbers are not a very sensitive probe of possible in-medium effects of the $\mathrm{K}^{-}$in dense matter.

In summary, our investigations seems to indicate that one has to look for more subtle observables to pin down the possible in-medium effects for the antikaons in a less ambiguous way. One such observable might be well constituted by the $\mathrm{K}^{-}$flow pattern. This feature of the $\mathrm{K}^{-}$flow is demonstrated in figure 6. Without medium modifications and even for a modified cross section, the $\mathrm{K}^{-}$shows an antiflow. An attractive $\mathrm{K}^{-}$optical potential makes the $\mathrm{K}^{-}$to flow with the nucleons. We found 


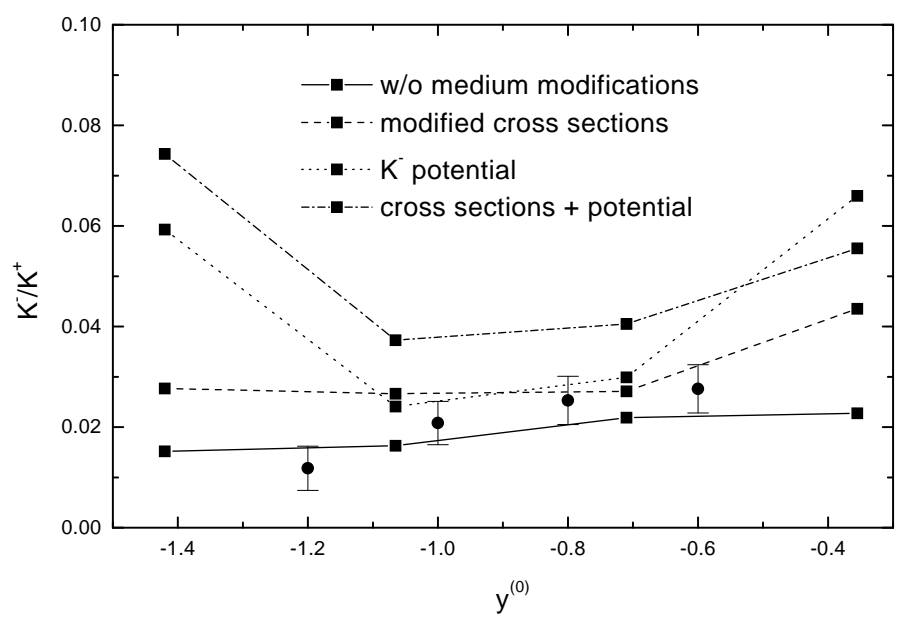

Figure 5. The ratio of produced $\mathrm{K}^{-} / \mathrm{K}^{+}$for $\mathrm{Ru}+\mathrm{Ru}$ collisions at $1.69 \mathrm{AGeV}$ for $b<4 \mathrm{fm}$ as a function of beam rapidity $y^{(0)}=y_{\text {beam }} / y_{\text {CMS }}-1$ (taken from [6]) in comparison with the data of the FOPI collaboration [15].

that only an attractive potential for the $\mathrm{K}^{-}$switches from an antiflow to a flow of the $\mathrm{K}^{-}$. The inclusion of temperature effects will also result in an antiflow pattern. Therefore, a measurement of the flow of the $\mathrm{K}^{-}$might be better suited to extract the attractive optical potential of the $\mathrm{K}^{-}$than the $\mathrm{K}^{-}$production rates.

\section{Hyperons in matter}

\subsection{Baryon-baryon interactions and strange dibaryons}

In the following we discuss the properties of hyperons in dense matter. The detailed study of $\Lambda$-hypernuclei gives important information about the underlying nucleon- $\Lambda$ interaction. Actually, the $\Lambda$ is the only hadron, besides the nucleons, where we know for sure the in-medium properties at normal nuclear matter density as it has been measured to be $U_{\Lambda}=-27 \mathrm{MeV}$, contrary to e.g. the kaons and antikaons. For the other hyperons the situation is less clear. No bound states of heavy $\Sigma$-hypernuclei have been found [18] (note that the only known $\Sigma$-hypernucleus ${ }_{\Sigma}^{4} \mathrm{He}$ is bound by virtue of an attractive isospin force [19]). The nucleon- $\Xi$ interaction as extracted from the few $\Xi$-hypernuclear events is attractive and gives a potential depth of $U_{\Xi}=-20$ to -25 $\mathrm{MeV}$ at $\rho_{0}$ 20]. Recent measurements of final state interactions of nucleons and $\Xi$ indicate a somewhat smaller value of $U_{\Xi}=-14 \mathrm{MeV}$ or less [21]. The $\Lambda \Lambda$ interaction is also found to be attractive in the available double $\Lambda$ hypernuclear data [22], even more attractive than the $\mathrm{N} \Lambda$ interaction. For the other hyperon-hyperon interactions $(\Lambda \Sigma, \Sigma \Sigma, \Lambda \Xi, \Sigma \Xi$ and $\Xi \Xi)$ nothing is known at present. Nevertheless, a strongly attractive hyperon-hyperon interaction can quite strongly effect the global properties of a neutron star. A third solution to the Tolman-Oppenheimer-Volkov equations can be found for highly attractive hyperon-hyperon potentials, which has extremely small radii of $6-8 \mathrm{~km}$ 23. Hence, these so called hyperstars have similar small radii as the ones obtained for kaon condensation in neutron stars. 


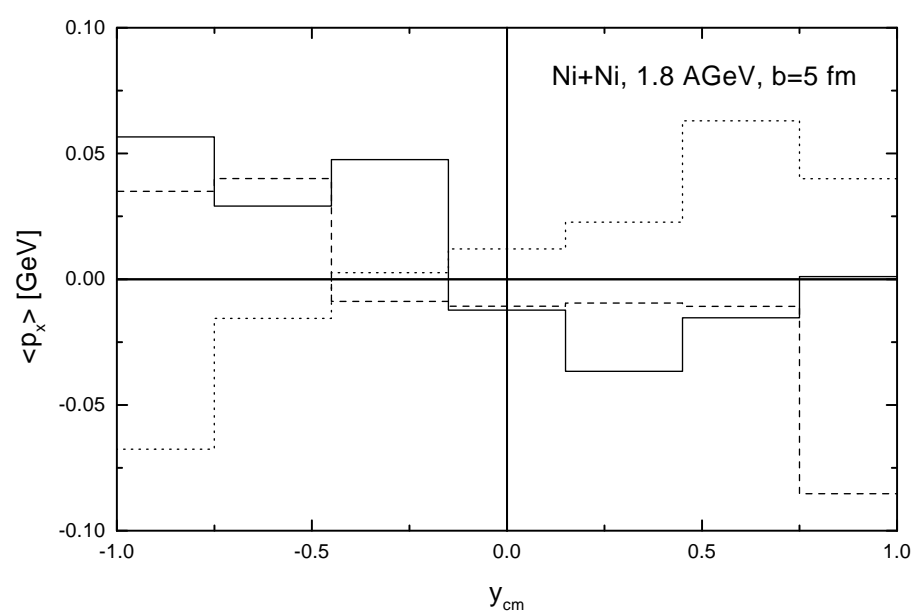

Figure 6. The flow of $\mathrm{K}^{-}$in a $\mathrm{Ni}+\mathrm{Ni}$ collision at $1.8 \mathrm{AGeV}$ and $b=3 \mathrm{fm}$ with and without medium modifications (taken from 6 6). The $\mathrm{K}^{-}$'s flow with the nucleons only for an attractive optical potential, all other cases studied give an antiflow for the $\mathrm{K}^{-}$.

Theoretically, the strength of the hyperon-hyperon interaction can not be deduced from general arguments. Model calculations predict that the hyperon-hyperon interaction is so attractive in certain channels that it leads to the existence of bound systems of two hyperons. The most recent Nijmegen model even suggests that states of $\Sigma \Xi$ and $\Xi \Xi$ dibaryons might be deeply bound [24].

\subsection{Weak decay patterns and signals in heavy-ion collisions}

Heavy-ion experiments are the only terrestrial source which can possibly shed light on the issue of the baryon-baryon interactions for hyperons. Dozens of hyperons can be produced in a single central heavy-ion collisions, sitting close in phase space. At freeze-out, pairs of hyperons can form and build a bound strange composite object. This strange dibaryon will then decay on the timescale of weak interactions. Here, we discuss possible signals, if such a bound strange dibaryon indeed exists. The weak decay of the strange dibaryon is modelled by using weak SU(3) symmetry for the parity-violating and parity-conserving amplitudes, whose parameters are fixed by the weak decay amplitudes of the octet hyperons [25]. There are two possible decays for the dibaryon: mesonic like in the case of free hyperon decay and nonmesonic without emitting a meson. The latter one is only possible in the medium and has been studied experimentally by the weak decay of medium to heavy $\Lambda$ hypernuclei. The standard approach to the nonmesonic decay of $\Lambda$ hypernuclei is the meson exchange model [26]. We extended the meson exchange model for the weak nonmesonic decay to the other hyperons taking into account only the intermediate pion and kaon exchanges 25. The resulting decay lengths of different strange dibaryons from these model calculations are in the range of $c \tau=1-5 \mathrm{~cm}$, which are only slightly shorter than the ones for the free hyperons.

For the two- $\Lambda$ dibaryon, the branching ratio for the various decay channels are summarized in figure 1 . The dominant decay channel for small binding energies is the 


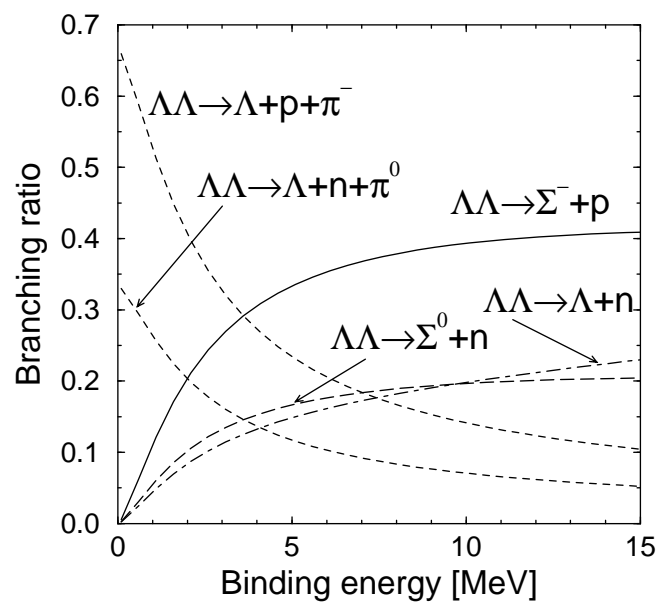

Figure 7. Branching ratios for the weak decay of a bound $(\Lambda \Lambda)$ dibaryon as a function of binding energy (taken from [25]).

free (mesonic) one, as the two $\Lambda$ 's are well separated. For larger binding energies, the dominant decay channel is $\Lambda \Lambda \rightarrow \Sigma^{-}+\mathrm{p}$, the same as for the $\mathrm{H}$ dibaryon [27] one is looking for in experiment E896 [28].

Figure 8 shows the case for a bound $\Xi^{0}$ p dibaryon. The $\Xi^{0} \mathrm{p}$ decays mainly into a $\Lambda$ and a proton for binding energies of only $1.5 \mathrm{MeV}$ and beyond. This decay can in principle be seen either in the invariant mass spectrum of $\Lambda$ 's and protons or directly by the decay topology. The decay of $\Xi^{0} \mathrm{p}$ dibaryon looks like the one for a normal $\Xi^{-}$ or $\Omega^{-}$hyperon, but instead of a meson a proton is leaving the first weak vertex.

Strange dibaryons can have quite exotic properties, as they can have negative charge while carrying a positive baryon number. There are three possible strange dibaryons which even have charge -2 : the $\Sigma^{-} \Sigma^{-}, \Sigma^{-} \Xi^{-}$and $\Xi^{-} \Xi^{-}$. Interestingly, all of these have been predicted to be bound in the recent version of the Nijmegen model [24]. The first candidate has only one possible decay which is mesonic: $\Sigma^{-} \Sigma^{-} \rightarrow \Sigma^{-}+\mathrm{n}+\pi^{-}$. As the matrix element of the mesonic decay is practically unchanged in the medium, we immediately know that the decay length of this object is $c \tau\left(\Sigma^{-} \Sigma^{-}\right)=c \tau\left(\Sigma^{-}\right) / 2=2.2 \mathrm{~cm}$. The other two candidates have mesonic as well as nonmesonic decay channels. But in all weak decays, there is a unique prong as a track with charge -2 suddenly splits up into two tracks with charge -1 . Such a weak decay is not possible for ordinary matter. It is in principle also sufficient to measure just the mass of an object with charge -2 as the strange dibaryons are lighter than an anti-helium nucleus.

The production rates of strange dibaryons have been estimated for $\mathrm{Au}+\mathrm{Au}$ collisions at BNL's Relativistic Heavy Ion Collider using RQMD2.4 with a wavefunction coalescence [25]. The rates vary between $10^{-2}$ to $10^{-4}$ per event depending on the strangeness content of the dibaryon. The rapidity distribution is rather flat, so that dibaryons are even produced at forward and backward rapidities. Hence, the production rates are reasonably high to search for these exotic states in heavy-ion collisions and open the door to the hitherto unexplored hyperon world. 


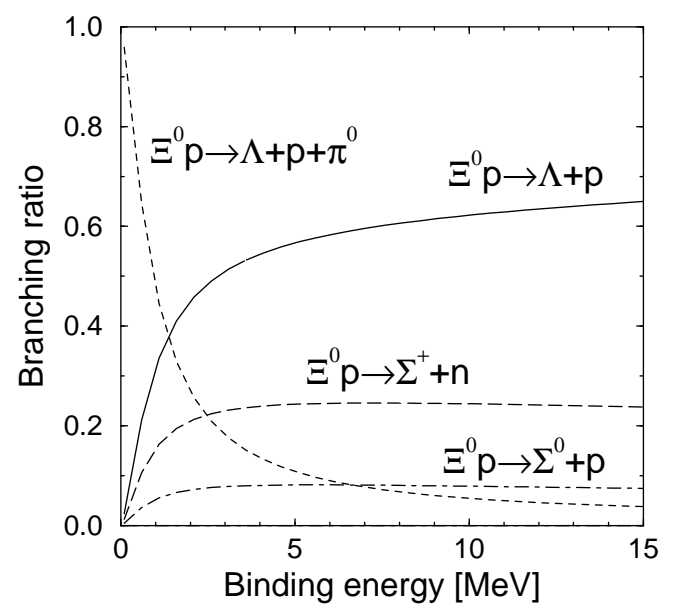

Figure 8. Branching ratios for the weak decay of a bound $\left(\Xi^{0} \mathrm{p}\right)$ dibaryon as a function of binding energy (taken from [25]).

\section{Acknowledgments}

JSB thanks the organizers of this conference for their kind support and RIKEN, Brookhaven National Laboratory and the U.S. Department of Energy for providing the facilities essential for the completion of this work.

\section{References}

[1] E. Friedman, A. Gal, and C. J. Batty, Phys. Lett. B 308, 6 (1993).

[2] E. Friedman, A. Gal, and C. J. Batty, Nucl. Phys. A579, 518 (1994).

[3] V. Koch, Phys. Lett. B 337, 7 (1994).

[4] G. Q. Li, C.-H. Lee, and G. E. Brown, Phys. Rev. Lett. 79, 5214 (1997).

[5] N. K. Glendenning and J. Schaffner-Bielich, Phys. Rev. Lett. 81, 4564 (1998).

[6] J. Schaffner-Bielich, V. Koch, and M. Effenberger, Nucl. Phys. A669, 153 (2000).

[7] M. Lutz, Phys. Lett. B 426, 12 (1998).

[8] A. Ramos and E. Oset, Nucl. Phys. A671, 481 (2000)

[9] L. Tolos, A. Ramos, A. Polls, and T. T. S. Kuo, nucl-th/0007042 (2000).

[10] see the contribution of Evgueni Krivoruchenko to these proceedings.

[11] A. Ohnishi, Y. Nara, and V. Koch, Phys. Rev. C 56, 2767 (1997).

[12] S. Teis, W. Cassing, M. Effenberger, A. Hombach, U. Mosel, and G. Wolf, Z. Phys. A 356, 421 (1997).

[13] M. Effenberger, E. L. Bratkovskaya, W. Cassing, and U. Mosel, Phys. Rev. C 60, 027601 (1999).

[14] R. Barth et al. (KaoS Collaboration), Phys. Rev. Lett. 78, 4007 (1997).

[15] N. Herrmann, Prog. Part. Nucl. Phys. 42, 187 (1999).

[16] J. Cleymans, H. Oeschler, and K. Redlich, Phys. Lett. B 485, 27 (2000).

[17] see the contribution of Helmut Oeschler to these proceedings.

[18] S. Bart et al., Phys. Rev. Lett. 83, 5238 (1999).

[19] T. Nagae et al., Phys. Rev. Lett. 80, 1605 (1998).

[20] C. B. Dover and A. Gal, Ann. Phys. (N.Y.) 146, 309 (1983).

[21] P. Khaustov et al., Phys. Rev. C 61, 054603 (2000).

[22] C. B. Dover, D. J. Millener, A. Gal, and D. H. Davis, Phys. Rey. C 44.1905 (1991).

[23] J. Schaffner-Bielich, M. Hanauske, H. Stöcker, and W. Greiner, astro-ph/0005490 (2000).

[24] V. G. J. Stoks and T. A. Rijken, Phys. Rev. C 59, 3009 (1999).

[25] J. Schaffner-Bielich, R. Mattiello, and H. Sorge, Phys. Rev. Lett. 84, (2000).

[26] A. Parreno, A. Ramos, and C. Bennhold, Phys. Rev. C 56, 339 (1997).

[27] J. F. Donoghue, E. Golowich, and B. R. Holstein, Phys. Rev. D 34, 3434 (1986).

[28] H. Caines et al. (E896 collaboration), Nucl. Phys. A661, 170c (1999) and these proceedings. 